\title{
A terahertz system using semi-large emitters: noise and performance characteristics
}

\author{
G Zhao, R N Schouten, N van der Valk, W Th Wenckebach \\ and P C M Planken \\ Department of Applied Physics, Faculty of Applied Sciences, Delft University of Technology, \\ Lorentzweg 1, 2628 CJ Delft, The Netherlands \\ E-mail: planken@tnw.tudelft.nl
}

Received 1 March 2002

Published 17 October 2002

Online at stacks.iop.org/PMB/47/3699

\begin{abstract}
We have built a relatively simple, highly efficient, terahertz (THz) emission and detection system centred around a 15 fs Ti:sapphire laser. In the system, $200 \mathrm{~mW}$ of laser power is focused on a $120 \mu \mathrm{m}$ diameter spot between two silverpaint electrodes on the surface of a semi-insulating GaAs crystal, kept at a temperature near $300 \mathrm{~K}$, biased with a $50 \mathrm{kHz}, \pm 400 \mathrm{~V}$ square wave. Using rapid delay scanning and lock-in detection at $50 \mathrm{kHz}$, we obtain probe laser quantum-noise limited signals using a standard electro-optic detection scheme with a $1 \mathrm{~mm}$ thick (110) oriented ZnTe crystal. The maximum THz-induced differential signal that we observe is $\triangle P / P=7 \times 10^{-3}$, corresponding to a $\mathrm{THz}$ peak amplitude of $95 \mathrm{~V} \mathrm{~cm}^{-1}$. The $\mathrm{THz}$ average power was measured to be about $40 \mu \mathrm{W}$, to our knowledge the highest power reported so far generated with Ti:sapphire oscillators as a pump source. The system uses off-the-shelf electronics and requires no microfabrication techniques.
\end{abstract}

\section{Introduction}

Terahertz time-domain spectroscopy (THz-TDS) has gained widespread popularity in recent years due to the wide range of applications, both in science and technology (Grischkowsky et al 1990, Mittleman et al 1996, Harde et al 1997, Hunsche et al 1998). There is currently a great interest in the potential use of THz-TDS for biomedical imaging, in, for example, the early detection of skin cancer. One great advantage of $\mathrm{THz}$ radiation is that it is non-ionizing and extremely weak and thus, in all probability, totally harmless to humans. One of the problems faced by people engaged in $\mathrm{THz}$ imaging, however, is that for rapid image acquisition, a good dynamic range and signal-to-noise in the detected $\mathrm{THz}$ signals are required. This requires both excellent generation and detection techniques. Therefore, to gauge the performance of a $\mathrm{THz}$ generation and detection setup, and to facilitate a comparison between different setups, 
(a)

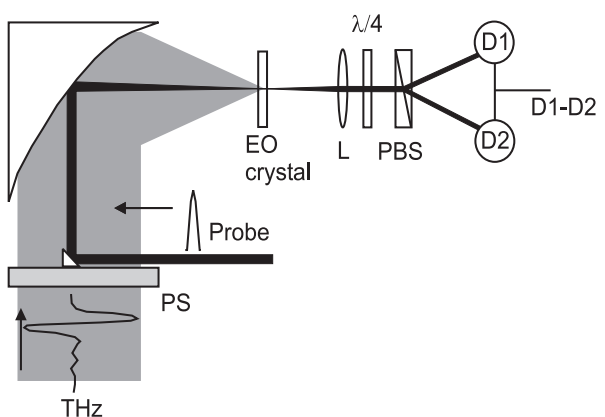

(b)

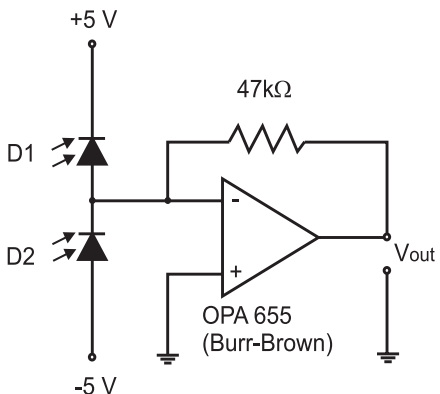

Figure 1. (a) The $\mathrm{THz}$ detection setup, with PS a $2 \mathrm{~mm}$ thick piece of polystyrene foam, $\mathrm{L}$ a lens, PBS a polarizing beamsplitter and $\lambda / 4$ a quarter-wave plate. Note the small triangle on the polystyrene plate representing the gold-coated prism. (b) Schematics of the differential detector. D1 and D2 are photodiodes of type BPW34 from Siemens.

a noise analysis is essential. Although such an analysis was given for a $\mathrm{THz}$ detection setup based on microfabricated antennas (van Exter and Grischkowsky 1990), a similar analysis is lacking for electro-optic detection.

Here, using an extensive noise analysis, we show that the combination of electrooptic detection and simple, semi-large aperture, biased, semi-insulating GaAs (SI-GaAs) emitters, provides an excellent $\mathrm{THz}$ generation/detection system, which can be used for $\mathrm{THz}$ spectroscopy and imaging. Surprisingly, we find that biased SI-GaAs with simple, silverpaint electrodes can be a very powerful $\mathrm{THz}$ emitter, surpassing the best high power emitters mentioned in the literature for systems using Ti:sapphire oscillators. Using a room-temperature pyroelectric detector for absolute power measurements, we find that approximately $40 \mu \mathrm{W}$ of $\mathrm{THz}$ power can be generated. This number is supported by the value of $95 \mathrm{~V} \mathrm{~cm}-1$ of the THz electric field measured in our electro-optic detection setup. Finally, a comparison with calculations demonstrates that our detection limit is determined by probe laser quantum noise only.

\section{Experimental details}

The femtosecond laser used in our experiments is from Femtolasers Produktions, Austria. It generates $450 \mathrm{~mW}$ of average power, and produces $15 \mathrm{fs}$ pulses with a $72 \mathrm{MHz}$ repetition rate and a centre wavelength of $775 \mathrm{~nm}$. The emitter is a crystal of SI-GaAs (MCP Wafer Technology Ltd), which has a dark resistivity of more than $5 \times 10^{7} \Omega \mathrm{cm}$. On the surface of the semiconductor, two crescent-shaped silverpaint electrodes are placed with a smallest separation of $0.4 \mathrm{~mm}$. $200 \mathrm{~mW}$ of average laser power is used to illuminate the emitter. A $50 \mathrm{kHz}, \pm 400 \mathrm{~V}$ square wave ac bias voltage is applied to the electrodes. A silicon hyperhemispherical lens is glued on the back of the crystal to focus the, initially strongly divergent, emitted radiation in a more forward direction. The optical path of the vertically polarized $\mathrm{THz}$ beam is shown in figure 1. After reflecting from several paraboloidal mirrors, the $\mathrm{THz}$ beam passes through a $2 \mathrm{~mm}$ thick piece of polystyrene foam. The polystyrene foam has a refractive index of 1.017 and an absorption coefficient of less than $1 \mathrm{~cm}^{-1}$ for frequencies below $4 \mathrm{THz}$, and is thus nearly completely transparent in this frequency range, while blocking any remaining near-infrared 'leaking' around the emitter (Zhao et al 2002). A small, 


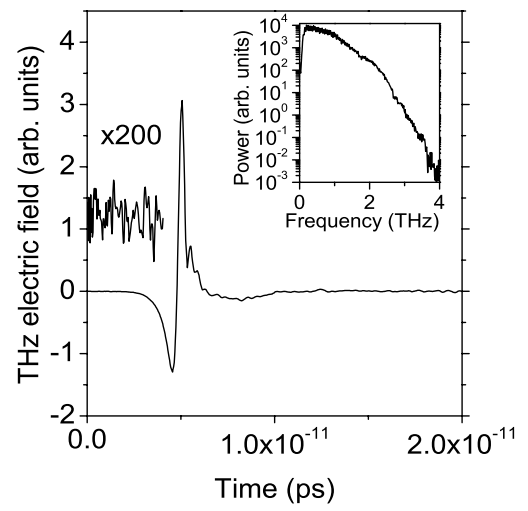

Figure 2. Measured THz electric field as a function of time, obtained using a $1 \mathrm{~mm}$ thick (110) oriented ZnTe detection crystal. This measurement corresponds to a total scan time of $20 \mathrm{~ms}$. A signal obtained without the presence of the THz electric field is magnified 200 times and plotted in the same figure. This signal is displaced vertically for clarity. The inset shows the power spectrum calculated from the time-domain signal.

gold-coated, right-angle prism is glued onto the foam. The prism is used to collinearly combine the THz pulse with a synchronized, time-delayed, horizontally polarized probe pulse. Both the $\mathrm{THz}$ beam and the probe beam are focused onto the detection crystal, using a $5 \mathrm{~cm}$ focal length, gold-coated paraboloidal mirror. The detection process is based on the standard electro-optic detection using a quarter-wave plate, a Wollaston prism and a home-built differential detector. The basic circuitry of this detector is shown in figure 1(b).

\section{Results}

In figure 2, we plot (top) the THz electric field, measured using a $1 \mathrm{~mm}$ thick (110) oriented ZnTe electro-optic crystal (Ingcrys Laser Systems Ltd). This electric-field scan was obtained in a total time of $20 \mathrm{~ms}$. We show the results of a measurement when no $\mathrm{THz}$ electric field is present, magnified by a factor of 200 , demonstrating the excellent dynamic range of this measurement.

In the inset of figure 2 we plot the power of the THz pulse as a function of frequency. From this figure it can be seen that the spectrum of the THz pulse peaks at roughly $0.5 \mathrm{THz}$. For this detection crystal, frequency components up to $4 \mathrm{THz}$ are detected. When a $0.1 \mathrm{~mm}$ thick (110) oriented GaP crystal is used, components up to $6 \mathrm{THz}$ are detected (not shown here) at the expense of a smaller signal amplitude.

Using $1 \mathrm{~mm}$ thick, (110) oriented ZnTe crystal, we measure a relative differential signal $\Delta P / P_{\text {probe }}=7 \times 10^{-3}$, where $\Delta P=P_{1}-P_{2}$ is the difference in optical power on the two photodiodes, and $P_{\text {probe }}=P_{1}+P_{2}$ is the total probe power. Based on this, we calculate a peak $\mathrm{THz}$ electric field of $95 \mathrm{~V} \mathrm{~cm}^{-1}$ (Planken et al 2001). With this crystal, we obtain a typical dynamic range of about 5000 for a 35 ps long $\mathrm{THz}$ electric-field scan in a time of $20 \mathrm{~ms}$. An independent measurement of the THz power is performed using a roomtemperature pyroelectric detector, consisting of a detector element (Eltec), without window or coating, in conjunction with a home-built amplifier. The detector was calibrated using the known power of a helium-neon laser. There is some uncertainty about the response of the detector head at far-infrared wavelengths but it is believed that the response is about $10 \%$ lower in the far-infrared. Using this assumption, we deduce an average $\mathrm{THz}$ power of 

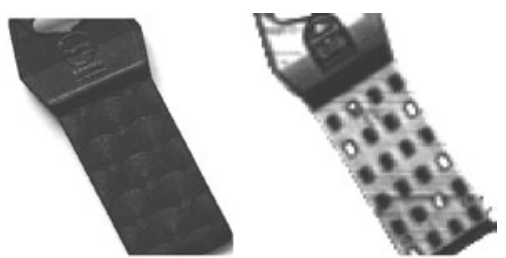

Figure 3. Picture (left) and THz peak-amplitude transmission image (right) of a plastic codekey.

$40 \mu \mathrm{W}$. To our knowledge, this value, supported by the large value of $\Delta P / P_{\text {probe }}$, represents the strongest $\mathrm{THz}$ signal reported in the literature using a Ti:sapphire oscillator as a pump source (Cai et al 1997, Wu and Zhang 1997, McLaughlin et al 2000, Heyman et al 2001, Andrews et al 2002).

An example of a THz image obtained with this setup is shown in figure 3 . The picture shows the $\mathrm{THz}$ peak-amplitude transmission image of a plastic codekey, measured by raster scanning the object through the focus of the $\mathrm{THz}$ beam. The $\mathrm{THz}$ image (picture on the right) clearly shows the presence (black squares) or absence (white squares) of small magnets contained by the plastic, whereas in the visible region of the spectrum (picture on the left), the plastic is completely opaque. This picture was taken in $8 \mathrm{~min}$, determined by the maximum speed of the $x y$-translation stage. We want to emphasize that, based on the high dynamic range observed in our $\mathrm{THz}$ measurements, orders of magnitude faster scanning speeds are, in principle, possible. For example, if it were technically possible to mechanically scan the codekey in $1 \mathrm{~s}$ instead of $8 \mathrm{~min}$, the predicted dynamic range per pixel would still be 227, more than sufficient for most applications. The noise properties of the detection setup determine to a large extent whether the signal-to-noise of the setup can be improved or not. The theoretical lower limit of the detector is the shot-noise (sn) limit. With $4.65 \mathrm{~mW}$ of average probe power on each photodiode, having a response of $0.49 \mathrm{~A} \mathrm{~W}^{-1}$, an average current of $2.28 \mathrm{~mA}$ is generated. The root-mean-square (rms) shot-noise current fluctuations, expressed in $\mathrm{A} \mathrm{Hz}^{-1 / 2}$, can be calculated according to

$$
\sqrt{\frac{\overline{\left(\Delta I_{\mathrm{sn}}\right)^{2}}}{B}}=\sqrt{2 q I_{0}}
$$

with $q$ the elementary charge, $I_{0}$ the current in the diodes and $B$ the measurement bandwidth in $\mathrm{Hz}$. We find a value of $\sqrt{\frac{\overline{\left(\Delta I_{\text {sn }}\right)^{2}}}{B}}=2.7 \times 10^{-11} \mathrm{~A} \mathrm{~Hz}^{-1 / 2}$ for each diode. For the two photodiodes combined, we obtain $\sqrt{\frac{2 \overline{\left(\Delta I_{\mathrm{sn}}\right)^{2}}}{B}}=3.82 \times 10^{-11} \mathrm{~A} \mathrm{~Hz}^{-1 / 2}$. What is observed in the experiment is the shot-noise voltage $V_{\mathrm{sn}}$ generated across the $47 \mathrm{k} \Omega$ resistor shown in figure $1(\mathrm{~b})$. We calculate $V_{\mathrm{sn}}=1.79 \times 10^{-6} \mathrm{~V} \mathrm{~Hz}^{-1 / 2}$. We plot this number as the upper horizontal dashed line in figure 4. In the same figure, we also plot the measured noise spectrum of this detector, using a Stanford Research Systems SR760 spectrum analyser, in the range between 1 and $100 \mathrm{kHz}$. Our THz signals are modulated and detected at $50 \mathrm{kHz}$ and this is therefore the range in which we have to compare the measured and the calculated noise. As seen from the figure, the agreement in this range is excellent, indicating that the detector truly operates in the shot-noise limit. In the same figure, we also plot the measured noise from the differential detector when there is no light on the photodiodes, together with a calculation of the thermal noise generated by the $47 \mathrm{k} \Omega$ resistor shown in figure 1 . The agreement between the measured and the calculated noise curves shows that without illumination, the noise is 


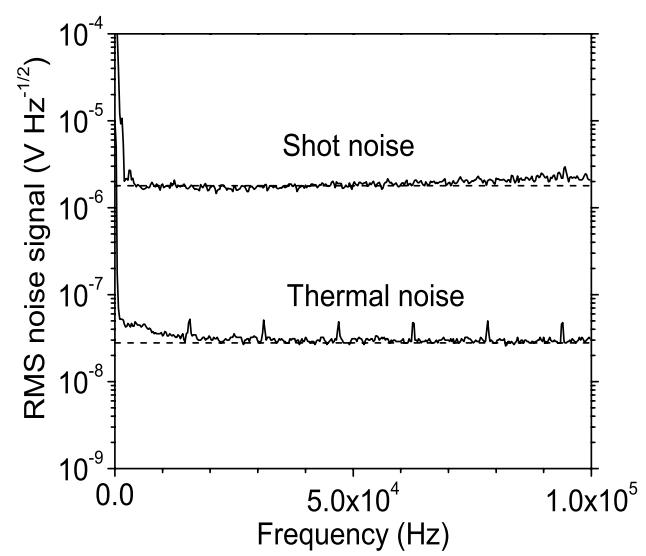

Figure 4. Top curves: measured (solid line) and calculated (dashed line) shot-noise spectra from our differential detector. Bottom curves: measured (solid line) and calculated (dashed line) thermal noise spectra from our differential detector.

mainly determined by thermal noise. The sharp spikes at regular intervals are an artefact caused by magnetic pick-up from the CRT of the spectrum analyser.

We define the noise-equivalent differential power (NEDP) as the minimum detectable probe power difference $\Delta P_{\mathrm{NEDP}}$, capable of generating a current equal to the rms shot-noise current. The smallest detectable relative power fluctuations can then also be expressed in terms of

$$
\frac{\Delta P_{\mathrm{NEDP}}}{\left(P_{\text {probe }} \sqrt{B}\right)}=\frac{\sqrt{2 \overline{\left(\Delta I_{\mathrm{sn}}\right)^{2}}}}{\left(2 I_{0} \sqrt{B}\right)} .
$$

In this equation, the term on the right-hand side constitutes the relative current fluctuations in which the shot-noise $\sqrt{2 \overline{\left(\Delta I_{\mathrm{sn}}\right)^{2}}}$ is the combined shot-noise of the two photodiodes. Equation (2) is evaluated by using the corresponding currents in the differential detector: an average current of $I_{0}=2.28 \mathrm{~mA}$ runs through each photodiode. This is the current generated by a power $\frac{1}{2} P_{\text {probe }}$. With $\sqrt{\frac{2\left(\overline{\left(I_{\mathrm{sn}}\right)^{2}}\right.}{B}}=3.82 \times 10^{-11} \mathrm{~A} \mathrm{~Hz}^{-1 / 2}$, we find $\Delta P_{\mathrm{NEDP}} /\left(P_{\text {probe }} \sqrt{B}\right)=$ $8.4 \times 10^{-9} \mathrm{~Hz}^{-1 / 2}$. A typical, $40 \mathrm{ps}$ long scan of a THz electric field obtained in $20 \mathrm{~ms}$ with a $20 \mathrm{kHz}$ bandwidth, gives $\Delta P_{\mathrm{NEDP}} /\left(P_{\text {probe }}\right)=1.2 \times 10^{-6}$.

Neglecting a small contribution from photodiode dark current, the photodiode shot-noise is the direct consequence of the quantized nature of the probe beam that causes carrier generation. The rms relative fluctuations of the number of photons in the probe beam, as would be measured in a spectrum analyser, using a detector with a $100 \%$ quantum efficiency and a bandwidth $B$, are given by (Haus 2000)

$$
\frac{\Delta N_{v}}{N_{\nu} \sqrt{B}}=\sqrt{\frac{4 \pi \hbar c}{P_{\text {probe }} \lambda}}
$$

with $\Delta N_{v}=\sqrt{\overline{N_{v}^{2}}}$ the probe beam photon fluctuations, $N_{v}$ the number of photons in the probe beam and $\lambda$ the wavelength of the probe. We find $\Delta N_{\nu} /\left(N_{v} \sqrt{B}\right)=7.3 \times 10^{-9} \mathrm{~Hz}^{-1 / 2}$. Using the real quantum efficiency of our detector of $\eta=80 \%$, the predicted value for the relative current fluctuations is then $\sqrt{\overline{2\left(\Delta I_{\mathrm{sn}}\right)^{2}}} /\left(2 I_{0} \sqrt{B}\right)=\Delta N_{\nu} /\left(N_{\nu} \sqrt{\eta B}\right)=8.2 \times 10^{-9} \mathrm{~Hz}^{-1 / 2}$, 
in agreement with the number $8.4 \times 10^{-9} \mathrm{~Hz}^{-1 / 2}$, found earlier. This demonstrates that probe laser quantum noise is the dominant noise source.

Finally, for completeness, we can calculate the lowest detectable $\mathrm{THz}$ power in our setup. The maximum peak THz electric field is $95 \mathrm{~V} \mathrm{~cm}^{-1}$, corresponding to a $\Delta P / P_{\text {probe }}=7 \times 10^{-3}$. Using the value $\Delta P_{\mathrm{NEDP}} /\left(P_{\text {probe }} \sqrt{B}\right)=8.4 \times 10^{-9} \mathrm{~Hz}^{-1 / 2}$ and assuming a reasonable measurement bandwidth of $1 \mathrm{~Hz}$, we obtain $\Delta P_{\mathrm{NEDP}} / P_{\text {probe }}=8.4 \times 10^{-9}$. We thus have a dynamic range of $8.3 \times 10^{5}$ in electric field, corresponding to a dynamic range of $6.9 \times 10^{11}$ in $\mathrm{THz}$ power. As the $\mathrm{THz}$ power associated with $95 \mathrm{~V} \mathrm{~cm}^{-1}$ is $40 \mu \mathrm{W}$, the minimum detectable $\mathrm{THz}$ power is $5.8 \times 10^{-17} \mathrm{~W}$, a number somewhat better than the number reported by van Exter and Grischkowsky (1990), using microfabricated antennas as THz detectors.

In conclusion, we have described a relatively simple $\mathrm{THz}$ generation and detection setup using semi-large aperture emitters and electro-optic detection. Our setup generates up to $40 \mu \mathrm{W}$ of average $\mathrm{THz}$ power with a dynamic range of $6.9 \times 10^{11}$, assuming a $1 \mathrm{~Hz}$ measurement bandwidth. To our knowledge, this constitutes the highest $\mathrm{THz}$ power generated by high repetition rate Ti:sapphire lasers to date. The setup may be ideally suited for biomedical $\mathrm{THz}$ imaging experiments which require short data acquisition times.

\section{Acknowledgment}

This work was performed as part of the EU TERAVISION programme (IST-1999-10154).

\section{References}

Andrews S R, Armitage A, Huggard P G and Hussain A 2002 Phys. Med. Biol. 47 3705-10 Cai Y, Brener I, Lopata J, Wynn J, Pfeiffer L and Federici J 1997 Appl. Phys. Lett. 712076 Grischkowsky D, Keiding S, van Exter M and Fattinger Ch 1990 J. Opt. Soc. Am. B 72006 Harde H, Cheville R A and Grischkowsky D 1997 J. Phys. Chem. A 1013646

Haus H A 2000 Electromagnetic Noise and Quantum Optical Measurements (Berlin: Springer)

Heyman J N, Neocleous P, Hebert D, Crowell P A, Mller T and Unterrainer K 2001 Phys. Rev. B 64085202

Hunsche S, Koch M, Brener I and Nuss M C 1998 Opt. Commun. 15022

McLaughlin R, Corchia A, Johnston M B, Chen Q, Ciesla C M, Arnone D D, Jones G A C, Linfield E H, Davies A G and Pepper M 2000 Appl. Phys. Lett. 7620

Mittleman D M, Jacobsen R H and Nuss M C 1996 IEEE J. Sel. Top. Quantum Electron. 2679

Planken P C M, Nienhuys H-K, Bakker H J and Wenckebach W Th 2001 J. Opt. Soc. Am. B 18313

van Exter M and Grischkowsky D R 1990 IEEE Trans. Microw. Theory Tech. 381684

Wu Q and Zhang X-C 1997 Appl. Phys. Lett. 701784

Zhao G, ter Mors M, Wenckebach W Th and Planken P C M 2002 J. Opt. Soc. Am. B 191476 